\title{
Avaliação sensorial e estabilidade físico-química de um blend de laranja e cenoura
}

\author{
Sensorial evaluation and physical-chemical stability of a blend of orange and carrot
}

\author{
Ivanise Guilherme BRANCO ${ }^{1 *}$, Eliana Janet SANJINEZ-ARGANDOÑA ${ }^{1}$, \\ Melissa Massaroli da SILVA ${ }^{1}$, Talissa Martins de PAULA ${ }^{1}$
}

\begin{abstract}
Resumo
Neste trabalho foi elaborado um blend de suco de laranja e cenoura com diferentes teores de cenoura (5 e $25 \%$ ) e concentrado a diferentes teores de sólidos solúveis ( 15 e $30^{\circ} \mathrm{Brix}$ ). Foram realizadas análises físicas e químicas das matérias-primas e do blend com maior preferência sensorial, com a finalidade de caracterizar o produto e, principalmente, verificar as alterações do ácido ascórbico e dos carotenóides totais após o processo de concentração dos blends. Também foi investigada a estabilidade física e química do blend durante sessenta dias. O teste de ordenação-preferência mostrou que a formulação do blend com $5 \%$ de cenoura e $15{ }^{\circ}$ Brix de concentração foi a preferida pelos julgadores. O processo de concentração e o armazenamento, pelo período de 60 dias, acarretou na redução significativa dos conteúdos de ácido ascórbico e carotenóides totais.
\end{abstract}

Palavras-chave: blend; análise sensorial; laranja; cenoura.

\begin{abstract}
In this work a "blend" of orange and carrot juice with different proportions of carrot ( 5 and $25 \%$ ) and concentrated to different degrees of soluble solids ( 15 and $30^{\circ}$ Brix) was developed. Physical and chemical analyses of the raw material and most sensorial preference "blend" were carried out with the main aim of characterizing the product. Special attention was paid to observe changes in the proportion of ascorbic acid and total carotenoides after the concentration juice process. The physical and chemical stability of the blend was also investigated during a period of sixty days. In the sensorial analysis the ordering preference test was applied. The results showed that the formulation with the most sensorial preference was that with $5 \%$ of carrot and concentration up to $15^{\circ}$ Brix. The concentration process and the storage, for the period of 60 days, caused a significant reduction of the contents of ascorbic acid and total carotene.

Keywords: blend; sensorial analysis; orange; carrot.
\end{abstract}

\section{Introdução}

Nos últimos anos, tem-se notado um aumento no consumo de frutas e vegetais em razão do potencial na prevenção de doenças cardiovasculares e câncer, já que estes alimentos são fontes de vitaminas antioxidantes ( $\mathrm{C}$ e E), compostos fenólicos e carotenos ${ }^{10}$. Conseqüentemente houve uma grande expansão da agroindústria de frutas e hortaliças, principalmente na indústria de sucos, a qual tem uma expressiva importância econômica no País. Atualmente o Brasil é o terceiro maior produtor de frutas, cuja produção superou os 38 milhões de toneladas em $2002^{12}$ e o primeiro na produção de laranjas, sendo que aproximadamente $72,5 \%$ destas frutas são utilizadas para produção de suco de laranja concentrado e congelado. $\mathrm{O}$ volume total produzido de suco de laranja concentrado congelado em 2002 foi aproximadamente 1.200.000 toneladas, fazendo com que ocupe a segunda posição entre os produtos comercializados no mercado internacional ${ }^{7}$. No entanto, a partir do início dos anos 90 o mercado de suco de laranja começou a apresentar mudanças, como o surgimento de novas formas de comercialização do produto, uma tendência à auto-suficiência e competitividade dos norte-americanos nos laranjais da Flórida, a implantação de um programa de estabilidade econômica no Brasil e os novos hábitos modernos, induzindo ao consumo de produtos naturais, fez crescer o consumo deste produto no mercado nacional ${ }^{19}$.

\footnotetext{
Recebido para publicação em 12/5/2005

Aceito para publicação em 24/1/2007 (001526)

Departamento de Engenharia de Alimentos,

Universidade Estadual do Centro-Oeste - UNICENTRO,

Rua Presidente Zacarias, 875, CEP 85010-990, Guarapuava - PR, Brasil

E-mail: ivanise@unicentro.br

*A quem a correspondência deve ser enviada
}

O hábito do consumo de sucos de frutas e hortaliças processados tem aumentado, motivado pela falta de tempo da população em preparar suco das frutas in natura, pela praticidade oferecida pelos produtos, substituição ao consumo de bebidas carbonatadas, devido ao seu valor nutritivo, e preocupação com o consumo de alimentos mais saudáveis ${ }^{18}$. Entre 2001 e 2004, o consumo de sucos, néctares e drinques de frutas industrializadas cresceu em média $14 \%$ ao ano ${ }^{6}$, sendo o Brasil o segundo maior mercado da América Latina com produção estimada em 1 bilhão de litros. Além disso, esse mercado cresce em proporções maiores que o de refrigerantes e contribui com mais de 1 bilhão de dólares para as exportações brasileiras ${ }^{22}$.

$\mathrm{Na}$ indústria brasileira, há uma diversidade de sabores de sucos de frutas, embora o maior destaque seja dado para o de laranja, devido ao seu sabor conhecido, ampla aceitabilidade e grande disponibilidade. A procura por sabores diversificados também é grande, o que tem levado as empresas a desenvolverem novos produtos para atender a demanda. Uma alternativa para a diversificação é a incorporação de produtos agrícolas com fontes importantes de princípios nutritivos e compostos naturais, como é o caso da cenoura, que, além de possuir grande quantidade de compostos de interesse alimentar como fibras, carotenóides e sais minerais, possui grande disponibilidade da matéria-prima a baixo custo ${ }^{5}$. No entanto, o suco puro desse vegetal apresenta preferência limitada, devido ao sabor relativamente fraco, necessitando da combinação com outro vegetal para que resulte em um produto com melhor qualidade sensorial. 
Portanto, neste trabalho, foi elaborado um blend de suco de laranja e cenoura. Chamam-se blends as misturas de sucos elaboradas com a finalidade de melhorar as características sensoriais dos componentes isolados. Os blends apresentam uma série de vantagens, como a possibilidade de combinação de diferentes aromas e sabores e a soma de componentes nutricionais ${ }^{23}$. A incorporação de cenoura ao suco de laranja agregará valor nutricional ao produto, já que a laranja tem elevado conteúdo de vitamina $\mathrm{C}$, e a cenoura, alto nível de carotenos, além de contribuir para o aumento do teor de fibras, cor e consistência do produto. O produto obtido poderá ser uma nova forma para inserção dessas vitaminas na dieta alimentar, como também um estímulo para o aumento do consumo de suco de laranja concentrado.

O processo de concentração de sucos pode influenciar na perda de alguns constituintes importantes do sabor e aroma. Por isso, torna-se necessário avaliar o efeito da concentração sobre a qualidade sensorial do blend estudado e as alterações nos teores de ácido ascórbico e carotenóides totais após processamento e armazenamento, já que estes são instáveis diante de luz, oxigênio e altas temperaturas.

Assim, o objetivo deste trabalho foi desenvolver um blend de laranja e cenoura, avaliar a aceitabilidade do produto por meio de testes sensoriais e verificar a estabilidade físico-química do produto obtido.

\section{Material e métodos}

\subsection{Matéria-prima}

As laranjas utilizadas no processamento do blend foram da variedade Pêra (Citrus sinensis), e as cenouras (Daucus carota L.), da variedade Nantes. As matérias-primas foram adquiridas no comércio local e provenientes de um único lote.

As laranjas e as cenouras foram armazenadas em geladeira, à temperatura de $5{ }^{\circ} \mathrm{C}$, até o momento de serem utilizadas no processamento.

\subsection{Métodos}

\section{Processamento das matérias-primas}

As laranjas foram lavadas com água clorada (10 ppm) e descascadas manualmente. A extração do suco foi feita com o auxílio de um extrator doméstico (marca Eletrolux). As cenouras, depois de lavadas com água clorada (10 ppm) e sua superfície externa retirada, foram cortadas e trituradas em um liquidificador doméstico (marca Walita) juntamente com o suco de laranja, com a finalidade de homogeneizar o produto.

\section{Formulação das misturas}

Nas formulações dos blends variou-se a proporção de cenoura (5 e $25 \%$ ) e estas foram concentradas até 15 e $30{ }^{\circ} \mathrm{Brix}$.

\section{Concentração das formulações}

As formulações foram concentradas em rotoevaporador (marca Fisatom) a 50 rpm e à temperatura de $70^{\circ} \mathrm{C}$. Os blends concentrados foram acondicionados em sacos de polietileno e armazenados a $-8{ }^{\circ} \mathrm{C}$ até o momento da análise sensorial e química.

\section{Avaliação sensorial}

$\mathrm{Na}$ avaliação sensorial das formulações, foi utilizado o teste de ordenação preferência, que tem como princípio a preferência que o consumidor demonstra sobre um produto em relação a outro.

Para a análise sensorial, os blends concentrados foram rediluídos em água destilada até a concentração próxima de $12{ }^{\circ} \mathrm{Brix}$, por este ser o valor médio de sólidos solúveis encontrado no suco de laranja natural. Aos blends rediluídos foram adicionados 0 ou $10 \%$ de sacarose com a finalidade de verificar a influência desta na aceitabilidade do produto. A análise sensorial foi realizada em duas etapas: primeiramente, dezessete provadores analisaram quatro amostras sem adição de sacarose; e, posteriormente, com adição de $10 \%$ de sacarose.

Os produtos elaborados das quatro formulações foram colocados em refrigerador por aproximadamente quatro horas, para equilíbrio da temperatura. As amostras foram servidas em copos plásticos brancos descartáveis, identificados por números de três dígitos, dispostos aleatoriamente. As avaliações foram feitas em cabines individuais, à temperatura ambiente, entre as 10-12 horas e as 14-17 horas, usando luz branca.

Os resultados foram avaliados estatisticamente por meio da tabela para o teste de ordenação de Newell e Mac Farlane que define o valor das diferenças críticas entre os totais de ordenação ao nível de 5\% (DUTCOSKY ${ }^{4}$ ).

\section{Caracterização das amostras}

Para a caracterização da laranja, cenoura e formulação com maior preferência na análise sensorial, foram realizadas as seguintes análises físico-químicas: $\mathrm{pH}$, determinado pelo método potenciométrico, conforme metodologia descrita pelas Normas do Instituto Adolfo Lutz ${ }^{13}$; acidez total titulável, segundo método oficial da $\mathrm{AOAC}^{1}$; sólidos solúveis, medidos diretamente em refratômetro; ácido ascórbico, segundo método oficial da $\mathrm{AOAC}^{1}$, modificado por BENASSI e ANTUNES ${ }^{2}$; carotenóides totais, determinados utilizando-se o método que se baseia na extração de carotenóides por acetona, armazenagem em éter de petróleo e leitura em espectrofotômetro, descrito por $\mathrm{SA}^{25}$; açúcares redutores e totais, conforme método de LaneEynon, baseado na redução de cobre pelos grupos redutores de açúcares $^{24}$.

\section{Resultados e discussão}

\subsection{Caracterização física e química das matérias-primas}

A caracterização física e química da laranja e da cenoura in natura encontra-se na Tabela 1.

Comparando-se os resultados da Tabela 1 aos obtidos por ZANGELMI et al. ${ }^{30}$, observa-se que o $\mathrm{pH}$, acidez total, 
Tabela 1. Características físicas e químicas das matérias-primas.

\begin{tabular}{lcr}
\hline \multicolumn{1}{c}{ Análises } & Laranja & \multicolumn{1}{c}{ Cenoura } \\
\hline Sólidos solúveis $\left({ }^{\circ} \mathrm{Brix}\right)$ & $10,0 \pm 0,000$ & $6,3 \pm 0,000$ \\
pH & $3,54 \pm 0,000$ & $5,92 \pm 0,000$ \\
Acidez total titulável $\left(\%{\left.\mathrm{v} \cdot \mathrm{v}^{-1}\right)}^{1,23 \pm 0,004}\right.$ & $0,80 \pm 0,000$ \\
Ácido ascórbico $\left(\mathrm{mg} .100 \mathrm{~g}^{-1}\right)$ & $57,62 \pm 0,648$ & $1,64 \pm 0,217$ \\
Açúcares redutores $(\%)$ & $7,58 \pm 0,660$ & $2,94 \pm 0,01$ \\
Açúcares totais $(\%)$ & $10,53 \pm 0,13$ & $3,21 \pm 0,09$ \\
Carotenóides Totais $\left(\mu \mathrm{g} \cdot \mathrm{g}^{-1}\right)$ & $31,9 \pm 0,22$ & $42,66 \pm 0,34$ \\
\hline
\end{tabular}

sólidos solúveis e ácido ascórbico do suco de laranja estão próximos aos da literatura, que foram $3,2,1,258,9,82{ }^{\circ} \mathrm{Brix} \mathrm{e}$ 60,73 mg. $100 \mathrm{~g}^{-1}$, respectivamente. O conteúdo de ácido ascórbico também foi próximo ao obtido por KABASAKALIS et al. ${ }^{15}$, que encontraram 52,3 mg. $100 \mathrm{~mL}^{-1}$, e ligeiramente superior ao obtido por LEE e COATES ${ }^{16}$, que obteve $40,6 \mathrm{mg} .100 \mathrm{~mL}^{-1}$.

Os resultados encontrados para teor de sólidos solúveis, acidez titulável, ácido ascórbico e açúcares redutores e totais para o suco de laranja estão dentro das faixas de variação citadas por TETRA PAK PROCESSING SYSTEM AB ${ }^{27}$ : para sólidos solúveis a faixa está entre 8,1 e $17,7^{\circ} \mathrm{Brix}$; para acidez, a variação está entre 0,58 e 1,73 g ácido cítrico. $100 \mathrm{~g}^{-1}$; o teor de ácido ascórbico oscila entre 23,5 e 76,0 mg. $100 \mathrm{~mL}^{-1}$ e os teores de açúcares redutores e totais variam, respectivamente, de 2,25 a 8,83 e de 6,23 a 14,3 g. $100 \mathrm{~g}^{-1}$.

Para a cenoura, os resultados de $\mathrm{pH}$ e teor de sólidos solúveis estão próximos aos encontrados por $\mathrm{BRANCO}^{3}$, que foram respectivamente, 5,83 e 6,0 ${ }^{\circ} \mathrm{Brix}$. Entretanto, o teor de ácido ascórbico foi inferior ao encontrado por $\mathrm{FRANCO}^{8}$, assim como o resultado de carotenóides totais, quando comparado com os obtidos por GODOY ${ }^{11}$, que obteve em média 53,8 $\mu \mathrm{g} \cdot \mathrm{g}^{-1}$ de $\beta$-caroteno e 13,4 $\mu$ g.g ${ }^{-1}$ de $\alpha$-caroteno, e QUINTEROS ${ }^{23}$, que encontrou 51,07 $\mu \mathrm{g}$. $\mathrm{g}^{-1}$ de $\beta$-caroteno e 13,4 $\mu \mathrm{g} . \mathrm{g}^{-1} \mathrm{de}$ $\alpha$-caroteno. Essas variações encontradas podem ser causadas por vários fatores como: o tipo de solo, fertilizantes utilizados, época e local de plantio, estocagem, comercialização, dentre outros. Além desses fatores, deve-se salientar que o método para quantificação de carotenóides é delicado, devido à grande instabilidade desse componente frente à luz.

\subsection{Análise sensorial}

Os resultados obtidos no teste de Ordenação pela Preferência dos consumidores são apresentados na Tabela 2. Pode-se observar que o blend com $5 \%$ de cenoura e $15{ }^{\circ} \mathrm{Brix}$ apresentou maior preferência, diferindo significativamente das amostras B e D e não diferindo da amostra C. A formulação com $25 \%$ de cenoura, $15{ }^{\circ}$ Brix e $0 \%$ de sacarose apresentou menor preferência, diferindo significativamente das amostras A e C, não diferindo da amostra D. Por esses dados pode-se verificar que amostras formuladas com a mesma proporção de cenoura e diferentes teores de sólidos solúveis (A-C e B-D) não apresentaram diferenças significativas em relação à preferência, mostrando que o principal fator para a aceitabilidade do produto é o teor de cenoura, já que a concentração final de sólidos solúveis, do blend concentrado, teve pouca influência.
Tabela 2. Somatória dos valores obtidos pelo teste de ordenaçãopreferência dos diferentes blends testados, com e sem adição de sacarose.

\begin{tabular}{|c|c|c|}
\hline Formulações & $0 \%$ sacarose & $10 \%$ sacarose \\
\hline $\begin{array}{l}\text { Amostra A - Blend com } 5 \% \text { de } \\
\text { cenoura e concentrado a } 15{ }^{\circ} \mathrm{Brix}\end{array}$ & $61^{\mathrm{a}}$ & $61^{a}$ \\
\hline $\begin{array}{l}\text { Amostra B - Blend com } 25 \% \text { de } \\
\text { cenoura e concentrado a } 15{ }^{\circ} \mathrm{Brix}\end{array}$ & $23^{\mathrm{b}}$ & $34^{\mathrm{bd}}$ \\
\hline $\begin{array}{l}\text { Amostra C - Blend com } 5 \% \text { de } \\
\text { cenoura e concentrado a } 30^{\circ} \mathrm{Brix}\end{array}$ & $54^{\mathrm{a}}$ & $50^{\mathrm{ad}}$ \\
\hline $\begin{array}{l}\text { Amostra D - Blend com } 25 \% \text { de } \\
\text { cenoura e concentrado a } 30^{\circ} \mathrm{Brix}\end{array}$ & $32^{\mathrm{b}}$ & $29^{\mathrm{bc}}$ \\
\hline
\end{tabular}

A formulação com $5 \%$ de cenoura, $15{ }^{\circ}$ Brix e $10 \%$ de sacarose também foi a mais preferida, como verificado na Tabela 2 , mostrando que a maior preferência é por formulações com baixa proporção de cenoura, independente da concentração de açúcar adicionado aos blends. Diferentemente ao observado nas formulações sem adição de açúcar, verifica-se que a formulação menos preferida é a amostra D. Essa baixa preferência pode ser devido à sacarose adicionada a uma formulação com alto teor de cenoura e sólidos solúveis, contribuindo para aumentar a consistência do produto obtido.

\subsection{Caracterização física e química dos blends}

A formulação do blend de laranja e cenoura mais aceita no teste de análise sensorial ( $15{ }^{\circ}$ Brix e $5 \%$ de cenoura) foi submetida às seguintes análises físico-químicas: sólidos solúveis, pH, acidez total, ácido ascórbico e carotenóides.

Os resultados obtidos da caracterização do blend natural (10 ${ }^{\circ} \mathrm{Brix}$ ) e concentrado ( $15^{\circ} \mathrm{Brix}$ ), estão apresentados na Tabela 3 .

Tabela 3. Caracterização física e química dos blends de laranja e cenoura natural ( $10{ }^{\circ} \mathrm{Brix}$ e $5 \%$ de cenoura) e concentrado ( $15{ }^{\circ} \mathrm{Brix}$ e $5 \%$ de cenoura).

\begin{tabular}{lcc}
\hline \multirow{1}{*}{ Análises } & \multicolumn{2}{c}{ Blend de laranja e cenoura } \\
\cline { 2 - 3 } & Natural & Concentrado \\
\hline Sólidos solúveis $\left({ }^{\circ} \mathrm{Brix}\right)$ & $10,0 \pm 0,0$ & $15,0 \pm 0,0$ \\
$\mathrm{pH}$ & $4,00 \pm 0,0$ & $4,01 \pm 0,0$ \\
Acidez total titulável $\left(\%{\left.\mathrm{v} \cdot \mathrm{v}^{-1}\right)}^{-1}\right.$ & $1,453 \pm 0,006$ & $1,40 \pm 0,005$ \\
Ácido ascórbico $\left(\mathrm{mg} \cdot 100 \mathrm{~g}^{-1}\right)$ & $60,192 \pm 0,35$ & $58,35 \pm 0,21$ \\
Carotenóides totais $\left(\mu \mathrm{g}^{-1}\right)$ & $51,52 \pm 0,31$ & $63,84 \pm 0,15$ \\
\hline
\end{tabular}

Pela Tabela 3, pode-se verificar que no processo de concentração dos blends não houve alteração do pH. Entretanto houve redução, não significativa, da acidez titulável. Esse resultado está de acordo com o obtido por SANDHU e BHATIA ${ }^{26}$, que também observaram não haver mudanças no pH e acidez durante a concentração de suco de laranja. Porém ZANGELMI et al. ${ }^{30}$ observaram um aumento significativo da acidez titulável com a concentração de suco de laranja.

Observou-se uma perda de aproximadamente 20,89 mg. $100 \mathrm{~g}^{-1}(34,94 \%)$ de ácido ascórbico no processo de concentração, valor este bem superior ao observado por ZANGELMI et al..$^{30}$, na concentração de suco de laranja de 9,82 
a 43,41 ${ }^{\circ}$ Brix. A perda de ácido ascórbico era esperada devido ao tratamento térmico durante a evaporação. SANDHU e BHATIA ${ }^{26}$ também verificaram que o ácido ascórbico foi altamente instável ao calor durante o processo de concentração de suco de laranja. No entanto, os mesmos autores verificaram que o teor de $\beta$-caroteno não foi afetado pelo processo de concentração.

A concentração de ácido ascórbico em suco de laranja e cenoura ( $80 \%$ de laranja e $20 \%$ de cenoura) sem tratamento foi reportado por TORREGROSA et al. ${ }^{28}$ que verificaram $27,11 \mathrm{mg} .100 \mathrm{~mL}^{-1}$, valor este inferior, mesmo quando comparado com a concentração de suco de laranja utilizada no presente trabalho. Essas variações no conteúdo de ácido ascórbico em suco de laranja podem ser devido à maturidade e variedade das laranjas, manejamento das frutas frescas, processamento e embalagem (citado por JOHNSTON; BOWLING ${ }^{14}$ ).

\subsection{Caracterização físico-química do blend de laranja e cenoura ( $5 \%$ de cenoura e concentrado a $15^{\circ}$ Brix) armazenado por 60 dias}

Com a finalidade de verificar as alterações no teor de sólidos solúveis, $\mathrm{pH}$, acidez total titulável, ácido ascórbico e carotenóides totais do blend concentrado, foram realizadas análises físico-químicas durante o período de armazenamento de 60 dias. As análises foram realizadas a cada 15 dias. Os resultados estão apresentados na Tabela 4.

Por meio da Tabela 4 e Figura 1, pode-se observar que o teor de sólidos solúveis se manteve constante durante os 60 dias de armazenamento, enquanto que o $\mathrm{pH}$ e acidez total sofreram uma pequena variação, mostrando que esses parâmetros foram ligeiramente afetados pelo tempo de armazenamento. Resultados semelhantes em relação aos sólidos solúveis e acidez total foram verificados por FREITAS et al. ${ }^{9}$ avaliando a estabilidade físico-química de suco de acerola submetido a tratamento térmico e armazenado a $28^{\circ} \mathrm{C}$ por 350 dias. No entanto, os mesmos autores observaram um discreto aumento do $\mathrm{pH}$ com o período de estocagem, atribuindo esse comportamento à perda de ácido cítrico durante o armazenamento. Resultados divergentes foram observados por PEDRÃO et al..$^{20}$, que não encontraram diferenças no teor de ácido ascórbico, ácido cítrico, sólidos solúveis e pH durante o armazenamento de suco de limão Tahiti congelado. Entretanto, a oxidação dos ácidos com o tempo e com as condições de armazenamento pode justificar a perda de acidez e conseqüente aumento do $\mathrm{pH}$.

Quanto aos dados encontrados para o ácido ascórbico e carotenóides totais, observou-se um decréscimo significativo dos conteúdos, com o tempo de armazenamento. Segundo TORREGROSA ${ }^{28}$, a concentração de ácido ascórbico em sucos de frutas diminui durante armazenamento e depende das

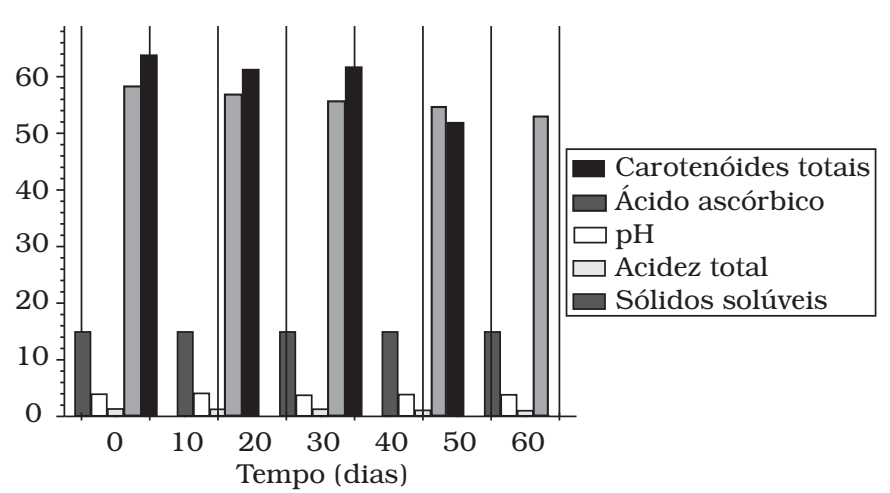

Figura 1. Comportamento dos parâmetros físico-químicos durante o período de armazenamento do blend ( $15^{\circ}$ Brix e $5 \%$ de cenoura).

condições de armazenamento, como temperatura, conteúdo de oxigênio e luz. TORREGGIANI e BERTOLO ${ }^{29}$ indicam que o ácido ascórbico é facilmente oxidado, principalmente em soluções aquosas e alcalinas. Com relação às perdas de carotenóides durante estocagem, estas podem ser associadas a uma exposição constante à luz por causa da natureza da embalagem. Oxidação de carotenóides é uma das principais causas para a perda de carotenóides durante o armazenamento, que é acelerado por luz, temperatura e presença de catalisadores metálicos (citado por FREITAS et al. ${ }^{9}$ ).

Nos sessenta dias de armazenamento, o blend reteve 90,91\% do ácido ascórbico e 78,79\% de carotenóides totais, indicando que o ácido ascórbico é mais estável com o congelamento do que os carotenóides totais. Trabalhos reportando perda de ácido ascórbico e/ou carotenóides totais foram verificados por LEE e COATES ${ }^{16}$, que observaram que o suco de laranja reteve $80,8 \%$ de ácido ascórbico durante o período de armazenamento de 24 meses a $-23{ }^{\circ} \mathrm{C}$; KABASAKALIS et al. ${ }^{15}$, que verificaram $28,9 \%$ de perda de ácido ascórbico, após 4 meses, em suco de laranja comercial longa vida armazenado em temperatura ambiente; FREITAS et al. ${ }^{9}$, que observaram uma redução de 45,12\% e 3,61\% nos conteúdos de ácido ascórbico e carotenóides totais, respectivamente, para suco de acerola previamente submetido a tratamento térmico e armazenado a $28{ }^{\circ} \mathrm{C}$ por 350 dias; e LOPES et al. ${ }^{17}$, que verificaram uma retenção de carotenóides de $86,24 \%$, trabalhando com polpa de pitanga congelada.

\section{Conclusões}

Com base nos resultados obtidos, pode-se concluir que:

- Houve uma significativa redução no conteúdo de ácido ascórbico e carotenóides totais durante o processo de

Tabela 4. Caracterização físico-química do blend (concentrado a $15{ }^{\circ}$ Brix e $5 \%$ de cenoura) armazenado por 60 dias.

\begin{tabular}{|c|c|c|c|c|c|}
\hline \multirow[t]{2}{*}{ Análises } & \multicolumn{5}{|c|}{ Tempo de Armazenamento (dias) } \\
\hline & 0 & 15 & 30 & 45 & 60 \\
\hline Sólidos solúveis ( ${ }^{\circ} \mathrm{Brix}$ ) & $15,0 \pm 0,000$ & $15,0 \pm 0,000$ & $15,0 \pm 0,000$ & $15,0 \pm 0,000$ & $15,0 \pm 0,000$ \\
\hline $\mathrm{pH}$ & $4,00 \pm 0,000$ & $4,14 \pm 0,000$ & $3,82 \pm 0,000$ & $3,93 \pm 0,000$ & $3,90 \pm 0,000$ \\
\hline Acidez total titulável (\%v.v $\left.{ }^{-1}\right)$ & $1,40 \pm 0,005$ & $1,34 \pm 0,002$ & $1,35 \pm 0,002$ & $1,15 \pm 0,001$ & $1,08 \pm 0,0004$ \\
\hline Ácido ascórbico (mg.100 g-1) & $58,35 \pm 0,21$ & $56,92 \pm 0,27$ & $55,73 \pm 0,11$ & $54,73 \pm 0,46$ & $53,05 \pm 0,34$ \\
\hline Carotenóides totais $\left(\mu \mathrm{g} \cdot \mathrm{g}^{-1}\right)$ & $63,84 \pm 0,15$ & $61,30 \pm 0,33$ & $61,88 \pm 0,78$ & $51,91 \pm 0,45$ & $50,30 \pm 0,26$ \\
\hline
\end{tabular}


concentração do suco de laranja com $5 \%$ de cenoura e $15{ }^{\circ}$ Brix em conseqüência do tratamento térmico;

- O armazenamento durante 60 dias praticamente não afetou as propriedades físico-químicas do suco de laranja com $5 \%$ de cenoura e concentrado a $15{ }^{\circ} \mathrm{Brix}$. As perdas de ácido ascórbico durante o período de armazenamento foram menores que $9,1 \%$, porém foi verificada perda significativa do teor de carotenóides totais $(21,2 \%)$; e

- O teste sensorial de ordenação mostrou que a formulação com $5 \%$ de cenoura e concentração do produto a $15{ }^{\circ} \mathrm{Brix}$, independente do teor de sacarose adicionado, foi a de maior preferência pelo consumidor. A formulação com maior teor de cenoura foi a menos preferida por apresentar alto teor de fibras insolúveis, gerando consistência indesejável.

\section{Referências bibliográficas}

1. ASSOCIATION OF OFFICIAL ANALYTICAL CHEMISTS- AOAC. Official Methods of Analysis. Washington, 14 ${ }^{\circ} \mathrm{ed} ., 1984$. $1094 \mathrm{p}$.

2. BENASSI, M. T.; ANTUNES, A. J. A comparasion of metaphosphoric and oxalic acids as extractant solutions for the determination of vitamin $\mathrm{C}$ in selected vegetables. Arquivos de Biologia e Tecnologia, v. 31, n. 4, p. 507-513, 1988.

3. BRANCO, I. G. Estudo do Comportamento Reológico de Misturas Ternárias com Sucos de Manga, Laranja e Cenoura. 2001. 140 f. Dissertação (Doutorado em Engenharia de Alimentos) - Faculdade de Engenharia de Alimentos, Universidade Estadual de Campinas, Campinas, 2001.

4. DUTCOSKY, S. D. Análise Sensorial de Alimentos. Curitiba: Champagnat, 1996. 123 p.

5. EMBRAPA. Empresa Brasileira de Pesquisas Agropecuárias. Hortaliças. Disponível em:<http://embrapa.br/linhas_de_acao/ alimentos/hortalicas/index_html/mostra_documento $>$. Acesso em 21 março 2005 .

6. EUGÊNIO, J. Alta pressão para conservação de sucos de frutas tropicais. Disponível em: <http://www.embrapa.br/ noticias/banco_de_noticias/folder.2006/folder noticia.200604-03.3722359657/noticia.2006-04-21.8447570502/mostra noticia >. Acesso em 13 setembro 2006.

7. FAO. Food and Agriculture Organization. Disponível em: $<\mathrm{http}: / /$ www.fao.org >. Acesso em 2 abril 2005.

8. FRANCO, G. Tabela de composição química dos alimentos. $9^{\circ}$ ed. Rio de Janeiro: Ed. Livraria Atheneu, 1999. 307 p.

9. FREITAS, C. A. S.; MAIA, G. A; SOUSA, P. H. M.; BRASIL, I. M.; PINHEIRO, A. M. Storage stability of acerola tropical fruit juice obtained by hot fill method. International Journal of Food Science and Technology, v. 41, n. 10, p. 1216-1221, 2006.

10. GARDNER, P. T.; WHITE, T. A. C.; MCPHAIL, D. B.; DUTHIE, G. G. The relative contributions of vitamin $\mathrm{C}$, carotenoids and phenolics to the antioxidant potential of fruits juices. Food Chemistry, v. 68 , n. 4, p. 471-474, 2000.

11. GODOY, H. T. Estudo de carotenóides e pró-vitamina A em alimentos. 1993. 185 f. Dissertação (Doutorado em Ciência de Alimentos) - Faculdade de Engenharia de Alimentos, Universidade Estadual de Campinas, Campinas, 1993.

12. IBRAF. Instituto Brasileiro de Frutas. Disponível em: $<\mathrm{http}: / /$ www.ibraf.org.br/x-no/iol/a7n43/inf43mail.htm >. Acesso em 15 outubro, 2006.
13. INSTITUTO ADOLFO LUTZ. Normas analíticas do Instituto Adolfo Lutz - Métodos químicos e físicos para análise de alimentos. São Paulo, $3^{\mathrm{a}}$ ed., 1985.

14. JOHNSTON, C. S.; BOWLING, D. L. Stability of ascorbic acid in commercially available orange juices. Journal of the American Dietetic Association, v. 102, n. 4, p. 525-529, 2002.

15. KABASAKALIS, V.; SIOPIDOU, D.; MOSHATOU, E. Ascorbic acid content of commercial fruit juices and its rate of loss upon storage. Food Chemistry, v. 70, n. 3, p. 325-328, 2000.

16. LEE, H. S.; COATES, G. A. Vitamin C in frozen, fresh squeezed, unpasteurized, polyethylene-bottled orange juice: a storage study. Food Chemistry, v. 65, n. 2, p. 165-168, 1999.

17. LOPES, A. S.; MATTIETTO, R. A.; MENEZES, H. C. Estabilidade da polpa de pitanga sob congelamento. Ciência e Tecnologia de Alimentos, Campinas (SP), v. 25, n. 3, jul./set., 2005.

18. MATSUURA, F. C. A. U.; ROLIM, R. Avaliação da adição de suco de acerola em suco de abacaxi visando à produção de um "Blend" com alto teor de vitamina C. Revista Brasileira de Fruticultura, Jaboticabal (SP), v. 24, n. 1, abr., 2002.

19. NETO, R. da S. C.; FARIA, J. de A. F. Fatores que influem na qualidade do suco de laranja. Ciência e Tecnologia de Alimentos, Campinas (SP), v. 19, n. 1, jan./abr., 1999.

20. PEDRÃO, M. R.; BALEIA, A.; MODESTA, R. C. D.; PRUDÊNCIOFERREIRA, S. H. Estabilidade físico-química e sensorial do suco de limão Tahiti natural e adoçado, congelado. Ciência e Tecnologia de Alimentos, Campinas (SP), v. 19, n. 2, mai./aug., 1999.

21. POLYDERA, A. C.; STOFOROS, N. G.; TAOUKIS, P. S. Quality degradation kinetics of pasteurised and high pressure processed fresh Navel orange juice: Nutritional parameters and shelf life. Innovative Food Science and Emerging Technologies, v. 6, n. 1, p. 1-9, 2005.

22. PORTAL DO EXPORTADOR: Disponível em: < http://www portaldoexportador.gov.br/Dinamica.asp?CodigoDaPublicacao $=1951 \&$ CaminhoTemplate $=$ noticiaportal. asp $>$. Acesso em 12 setembro 2006.

23. GUINTEROS, E. T. T. Processamento e Estabilidade de Néctares de Acerola-Cenoura. 1995. 96 f. Dissertação (Mestrado em Tecnologia de Alimentos) - Faculdade de Engenharia de Alimentos, Universidade Estadual de Campinas, Campinas, 1995.

24. RANGANNA, S. Manual of Analysis of Fruit and Vegetables Products. New Delhi: Tata McGraw - Hill Publishing Company Limited, 1977. $634 \mathrm{p}$.

25. SÁ, M. C. Carotenóides em alimentos preparados para consumo: Comparação de análise direta e cálculo pelos dados de retenção. 2001. 121 f. Dissertação (Mestre em Ciência de Alimentos) - Faculdade de Engenharia de Alimentos, Universidade Estadual de Campinas, Campinas, 2001.

26. SANDHU, K. S.; BHATIA, B. S. Physico-chemical changes during preparation of fruit juice concentrate. Journal of Food Science and Technology, v. 22, n. 3, p. 202-206, 1985.

27. TETRA PAK PROCESSING SYSTEMS AB. The Orange Book. Sweden: Ruter Press, 1998.

28. TORREGROSA, F.; ESTEVE, M. J.; FRÍGOLA, A.; CORTÉS, C. Ascorbic acid stability during refrigerated storage of orange-carrot juice treated by high pulsed electric field and comparasion with pasteurized juice. Journal of Food Engineering, v. 69, n. 1, p. 31-40, 2005. 
29. TORREGGIANI, D.; BERTOLO, G. Osmotic pre-treatments in fruit processing: chemical, physical and structure effects. Journal of Food Engineering, v. 49, n. 2-3, p. 247-253, 2001.

30. ZANGElmi, A. C. B.; SGARBIERI, V. C.; GARRUTti, R. S.; RODRIGUEZ, O.; VERLÊNGIA, F.; MENEZES, T. J. B.;
JORGE, J. P. N.; FIGUEIREDO, I. B.; MARSAIOLI JR, A. Estudo comparativo de diversas variedades paulistas de laranja na produção de suco concentrado. Coletânia do Instituto de Tecnologia de Alimentos, v. 1, p. 323-363,1966. 